\title{
SEPARATION OF AQUEOUS ISOPROPANOL THROUGH CHITOSAN/POLY(VINYL ALCOHOL) BLENDED MEMBRANES BY PERVAPORATION
}

\author{
S. H. Tan ${ }^{1}$, A. L. Ahmad ${ }^{1 *}$, M. G. Mohd. Nawawi ${ }^{2}$ and H. Hassan ${ }^{2}$ \\ ${ }^{1}$ School of Chemical Engineering, Universiti Sains Malaysia, Engineering Campus, \\ 14300 Nibong Tebal, Pulau Pinang, Malaysia. \\ ${ }^{2}$ Department of Chemical Engineering, Faculty of Chemical \& Natural Resources Engineering, \\ Universiti Teknologi Malaysia, 81310 UTM Skudai, Johor Bahru, Malaysia. \\ *chlatif@eng.usm.my
}

\begin{abstract}
Blend membranes consisting of chitosan/poly (vinyl alcohol) (CS/PVA) were prepared from a solution casting method and characterized via the pervaporation separation of isopropanol-water mixtures. The solution of pure chitosan was blended with poly(vinyl alcohol) at different compositions. The miscibility of the blended polymers was determined. Criteria for miscibility was based on the clarity of the blend and the transparency of the membrane obtained. The effects of feed concentration and chitosan content in membrane on the permeation flux and separation factor were also investigated. For the dehydration of 90 wt.\% of isopropanol-water mixtures the performance of the blend ratio of 30:70 (CS:PVA) exhibited the highest separation factor. It was shown that under the same operating conditions, the pervaporation separation index (PSI) for homogeneous membranes were higher than that of blended membranes for the entire feed concentrations.
\end{abstract}

Key Words: Blend, Chitosan, Poly (vinyl alcohol), Miscibility, Pervaporation.

\section{INTRODUCTION}

Pervaporation is a membrane process for separating miscible liquid where a liquid mixture is directly brought into contact with a semipermeable membrane and the permeate is removed as a vapour either by creating a vacuum condition or employing a carrier gas on the permeate side as shown in Fig. 1.

Pervaporation differs from other processes in that the membrane constitutes a barrier between a liquid in the liquid phase and the permeate in the vapour phase ${ }^{[1]}$. Pervaporation can be effectively used in the separation of azeotropic, close-boiling point, and heat sensitive mixtures ${ }^{[2]}$. According to Brüschke ${ }^{[3]}$ pervaporation processes can be utilized for different types of separation such as (i) extraction of water from organic liquids, (ii) extraction of volatile organic substances from aqueous streams and (iii) extraction of organic components from mixtures of organic liquids. Among the applications of the pervaporation technique, the dehydration of ethanol was the earliest and best developed practical process ${ }^{[4]}$.

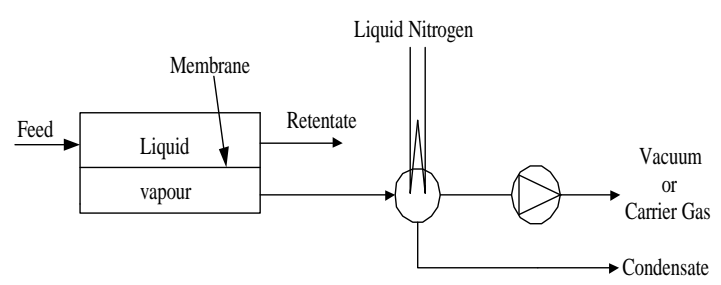

Fig. 1: Basic schematic diagram of pervaporation (Huang et al., 1993).

Pervaporation is one of the most developed membrane separation technology as it is capable of separating azeotropic mixtures or mixtures that have close boiling points. In 1982-83, Gesellschaft für Trenntechnik (GFT) Co., Germany, developed the first commercial pervaporation membrane by the use of poly (vinyl alcohol)-polyacrylonitrile composite membrane for the dehydration of alcohol solutions ${ }^{[5]}$.

Chitosan membrane is one of the hydrophilic membranes, which exhibited preferential permeation to water. Chitosan is a natural product derived from chitin, a polysaccharide found in the exoskeleton of shellfish like shrimp or crabs. Chitin is the second most abundant natural biopolymer after cellulose ${ }^{[6]}$. It was estimated that the worldwide annual recovery of chitin from processing of marine crustacean wastes was 37,300 metric tons ${ }^{[7]}$. Besides chitin, the shrimp shells do contain protein, and others inorganic compounds such as calcium carbonate, pigments and lipids. Although various methods have been cited in the literature to remove these impurities, the most common technique involves deproteinization and decalcification by using strong alkaline and acid.

Chitosan is a polymer of $[\beta-(1 \rightarrow 4)-2$-amino-2-deoxy-Dglucopyranose]. It is more amenable to the chemical, medical and industrial applications ${ }^{[8]}$, because of its solubility in various acidic solvents. In order to improve the pervaporation performances, the modified membranes have been studied. One of the modified membranes is blended membrane. When a mixture comprises of two or more polymers, which is not covalently bonded, it is called a polymer blend. The 
common techniques for preparing blends are melt mixing and solution casting ${ }^{[9]}$.

Homegeneous membrane is prepared from a single polymer material, while blended membrane is normally prepared from two different types of polymers. Polymer blending offers a convenient means to prepare materials with a wide range of properties. Through a suitable choice of a pair of polymer, polymer blends can often be tailor-made to have specific and desirable properties ${ }^{[10]}$. Blends of chitosan with poly(vinyl alcohol) ${ }^{[11,12]}$, silk fibroin ${ }^{[13]}$ and hydroxyethylcellulose ${ }^{[14]}$ had been reported.

The performance of the membrane used to separate a given binary A-B could be characterized by the experimental data, the total permeation flux, $J$, the amount of liquid that is transported through the membrane per unit area per unit of time. Separation factor $\alpha$ could be calculated by the following equation:

$\alpha_{\mathrm{A} / \mathrm{B}}=\frac{y_{A} / y_{B}}{x_{A} / x_{B}}$

where $y_{\mathrm{A}}$ and $y_{\mathrm{B}}$ are the weight fractions of components $\mathrm{A}$ and $\mathrm{B}$ in the permeate and $x_{\mathrm{A}}$ and $x_{\mathrm{B}}$ are the weight fractions of the components $\mathrm{A}$ and $\mathrm{B}$ in the feed. Component $\mathrm{A}$ is the more preferentially permeating component in the $\mathrm{A} / \mathrm{B}$ mixture.

From the practical point of view, membranes must have a high permeation flux together with large separation factor. However, in the actual pervaporation process normally there is a trade-off between the separation factor and permeation flux where a high separation factor is accompanied by low flux and vice versa. Thus, a new formula called pervaporation separation index (PSI) has been used for measuring the separation ability of a membrane. It can be defined as the product of a

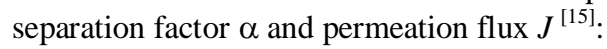

$$
\text { PSI }=J(\alpha-1)
$$

when $\alpha=1$, no separation occurs; a PSI of zero means either zero flux or zero separation.

The purpose of this study is to prepare chitosan blend with poly (vinyl alcohol) membranes. The pervaporation separations of the isopropanol-water mixtures through the blended membranes were conducted with isopropanol concentration in feed ranging from 10 to $95 \%$. The performance of membranes in pervaporation was evaluated by the permeation flux $(J)$, and the separation factor $(\alpha)$.

\section{MATERIALS}

Chitosan was prepared from domestic uncooked shrimp shells, which were collected from the hawkers nearby the campus. Poly (vinyl alcohol) polymer with the average Mw 124,000 - 186,000 was purchased from Aldrich Chemical Company (Germany). Reagent grade sodium hydroxide was purchased from All Chem (Sweden), ethanol and isopropanol from J. T. Baker (USA). Deionized distilled water was used in this study.

\section{EXPERIMENTAL PROCEDURE}

\subsection{Preparation of Chitin from Shrimp Shells}

The dried shells were cut into pieces with an average size of 2-6 mm. The shells were treated with $2-3$ molar of aqueous sodium hydroxide $(\mathrm{NaOH})$ to remove the protein content. The treatments were carried out at $80^{\circ}-90^{\circ} \mathrm{C}$ for 2 hours. Furthermore, they were treated in $2 \mathrm{M}$ aqueous hydrochloric acid $(\mathrm{HCl})$ at room temperature for 24 hours to remove the calcium from the shells ${ }^{[16]}$. Then the shells were washed with distilled water.

\subsection{Preparation of Chitosan from Chitin}

The chitin were further treated with $50 \mathrm{wt} \%$ of aqueous $\mathrm{NaOH}$ at temperature of $90^{\circ}-110^{\circ} \mathrm{C}$ for 3 hours in order to remove the acetyl group $\left(\mathrm{CH}_{3} \mathrm{CO}\right)$ from the chitin. The flakes obtained by the alkaline treatment were then washed with distilled water, dried under the sun for three hours and further dried up at room temperature. The products obtained are chitosan which were partly deacetylated. According to Robert ${ }^{[17]}$, the solubility of chitosan in dilute aqueous acids is obtained at an extent of deacetylation of $\sim 60 \%$ or above.

\subsection{Preparation of Homogeneous Chitosan Membranes}

A preweighed quantity of chitosan were first dissolved in 10 wt. $\%$ aqueous acetic acid solution at room temperature and stirred for 24 hours to produce a casting solution consisting of 2 wt.\% chitosan. The chitosan aqueous solution was filtered to remove any impurities and undissolved particles to produce a clear homogeneous casting solution by using a vacuum pump. The solution was allowed to settle down for about $3-4$ hours and the resulting casting solutions were cast onto a petri dish, allowing the casting solution to evaporate at room temperature for 48 hours. The formed membranes were treated in $3 \mathrm{wt} . \% \mathrm{NaOH}$ solution containing $47 \mathrm{wt}$. $\%$ ethanol, and $50 \mathrm{wt} . \%$ of distilled water for 24 hours at room temperature. The membranes were then washed thoroughly with distilled water to remove excess $\mathrm{NaOH}$ before being peeled off from the plate and dried at room temperature.

\subsection{Preparation of Blended membranes}

The solution casting method was used in preparation of chitosan/poly (vinyl alcohol) blends, in which both chitosan and poly (vinyl alcohol) homopolymers were separately dissolved in the respective solvent. Chitosan solutions were prepared by dissolving preweighed quantities of chitosan in $10 \mathrm{wt} \%$ aqueous acetic acid solution and stirring them at room temperature for 24 hours. Poly(vinyl alcohol) solutions were prepared by dissolving preweighed quantities of dry poly (vinyl alcohol) powder in deionized distilled water and heating it at $90^{\circ} \mathrm{C}$ for 6 hours. The casting solutions were prepared uniformly by mixing together the two solutions with given ratios. The casting solutions were 
cast onto petri dishes and dried at room temperature for 48 hours. The membranes were treated in 3 wt. \% $\mathrm{NaOH}$ solution containing 50 wt. \% ethanol solution for 24 hours at room temperature before being peeled off from the plate.

\subsection{Experimental Test Rig}

The pervaporation test rig is shown in Fig. 2. The main component of the pervaporation test rig is the membrane cell. The flat sheet membrane cell is fabricated from 316 stainless steel. The membrane cell consists of two portions namely, top and bottom. The top portion was equipped with two openings which were the inlet and outlet of the feed mixtures. Two porous stainless steel plates coated with teflon with the pores size of $30 \mu \mathrm{m}$, thickness of $1 \mathrm{~mm}$ and diameter of $90 \mathrm{~mm}$ were used to support the membrane. The two portions of the cell were clamped and tightly sealed using rubber O-ring. The effective area of the membrane cell was $52.81 \mathrm{~cm}^{2}$. The permeate vapour left the cell through the bottom side opening which was kept under vacuum by the vacuum pump to supply the necessary driving force for pervaporation. The feed mixture was stirred using Bibby stirrer and circulated from the feed tank to the permeation cell using Masterflex pristaltic pumps. A digital temperature controller was used to control the temperature of the feed mixtures. Furthermore, Swagelok control valve was used to control the downstream pressure. The permeate vapour was condensed in a cold trap by using liquid nitrogen. The condensed permeate was warmed up to ambient temperature and weighed to determine its flux. Three samples were taken for measuring of the flux and separation factor at every different feed concentration. The compositions of the permeate were determined by the Karl Fischer method ${ }^{[18]}$.

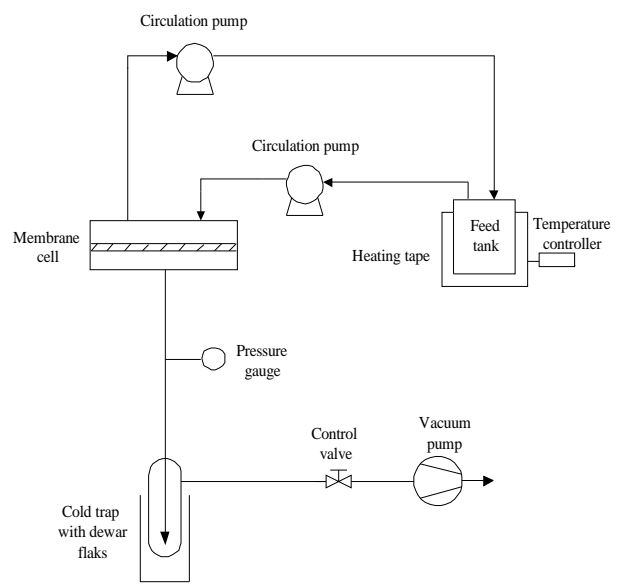

Fig. 2: Schematic diagram of the experimental rig for pervaporation process.

\section{RESULTS AND DISCUSSION}

Optical clarity is a good indication of miscibility of polymer blends. The blended solutions of chitosan/poly (vinyl alcohol) as well as the formed membranes were optically clear and transparent. These results reveal that the blends of chitosan and poly (vinyl alcohol) were compatible. Fig. 3 shows the pervaporation characteristics of blended membranes of 90 wt. \% of isopropanol in feed solution with different content of chitosan. The results show that the total permeation flux increases with an increase in the chitosan content. However the separation factor increased at an initial stage, it reached a miximum at chitosan content of 30 wt. $\%$ and then decreased. The best trade-off between the total permeation flux and its separation factor was achieved at $30 \mathrm{wt}$. \% of chitosan content in the blend ratio.

A plasticizing effect by the poly (vinyl alcohol) on the chitosan ${ }^{[11]}$ might have been expected. This resulted in the increase of the segmental mobility of the polymeric membrane as well as its free volume furthermore the diffusivity of permeating components would also be enhanced. Chanachai et al. ${ }^{[14]}$ studied the blend of chitosan/hydroxyethylcellulose for the pervaporation of ethanol-water mixtures also found a similar trend.

Fig. 4 shows the effect of blend ratio on the total and individual permeation fluxes at $90 \mathrm{wt} . \%$ of isopropanol in the feed. At chitosan content less than 45 wt. \%, high water permeation flux was accompanied by low isopropanol permeation flux. On the other hand, above 45 wt.\% of the chitosan content, a reverse trend occurred. Based on the above results, it is suggested that the polymer hydrophilicity was well balanced at the blend ratio of 30:70 (CS:PVA). Thus, the membrane at this particular blend ratio was chosen for further characterisation through pervaporation dehydration of isopropanol/water mixture with the effect of the feed concentrations.

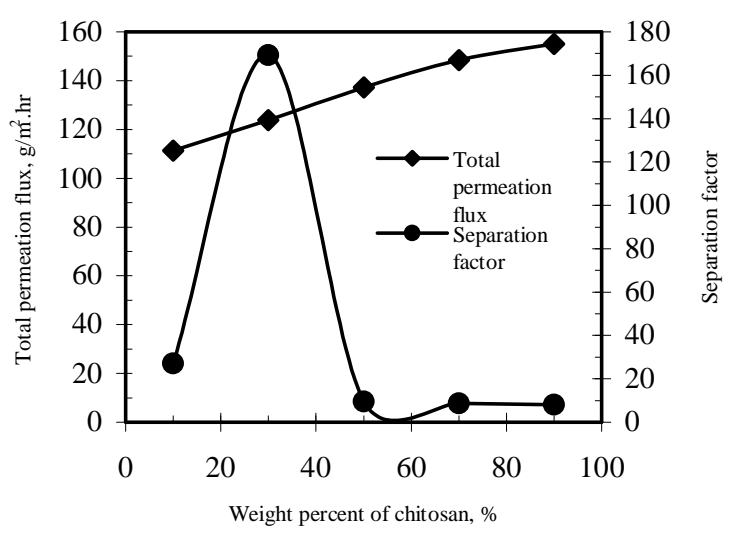

Fig. 3: Flux and separation factor at different $\%$ of chitosan. 


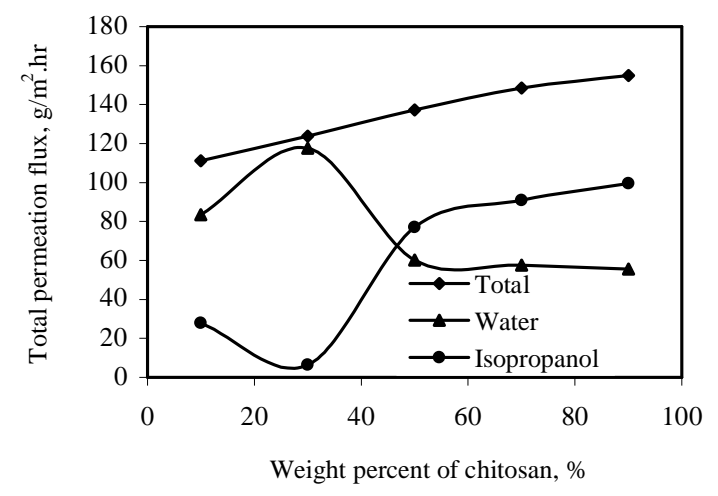

Fig. 4: Flux at different $\%$ of chitosan.

Figure 5 shows the total permeation flux and separation factor versus weight percent of isopropanol in feed solutions. The results showed that there was a trade-off between the total permeation flux and its separation factor for the entire feed solutions. The increase in the isopropanol concentration restricts the degree of swelling of the membranes, thus reducing the diffusivity of the permeating components.

Furthermore, the molecular size of isopropanol was bigger than that of water, so when the free volume of the membranes decrease at high concentration of isopropanol in feed, the permeation flux for water is higher than that of isopropanol. These could be the reasons why when the total permeation flux decreased, the separation factor increased and vice versa.

In order to measure the performance of the homogeneous and blended membranes, both the total permeation flux and separation factor have to be taken into consideration. Since there is a trade-off between the permeation flux $J$ and the separation factor $\alpha$, when one factor increases the other decreases. Thus the pervaporation separation index (PSI), which is defined as the product of $J$ and $(\alpha-1)$ has been widely used to evaluate the overall pervaporation membrane performance. The results are shown in Fig. 6. It was found that in terms of PSI, the CS/PVA membrane was higher than that of homogeneous membrane over the entire range of feed compositions.

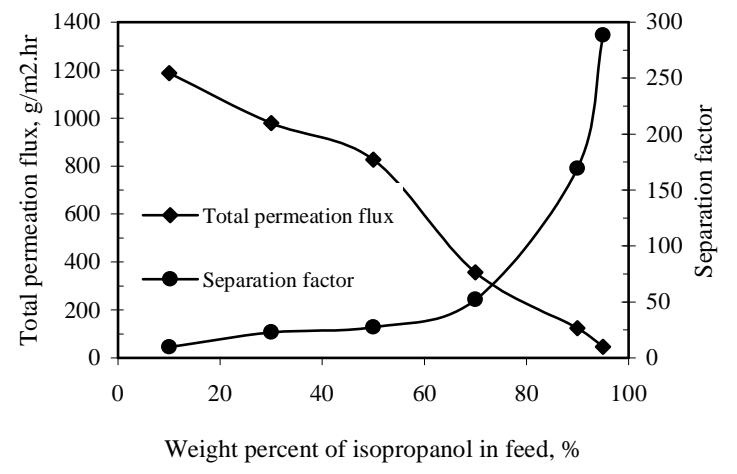

Fig. 5: The total permeation flux and separation factor at different $\%$ of isopropanol in feed.

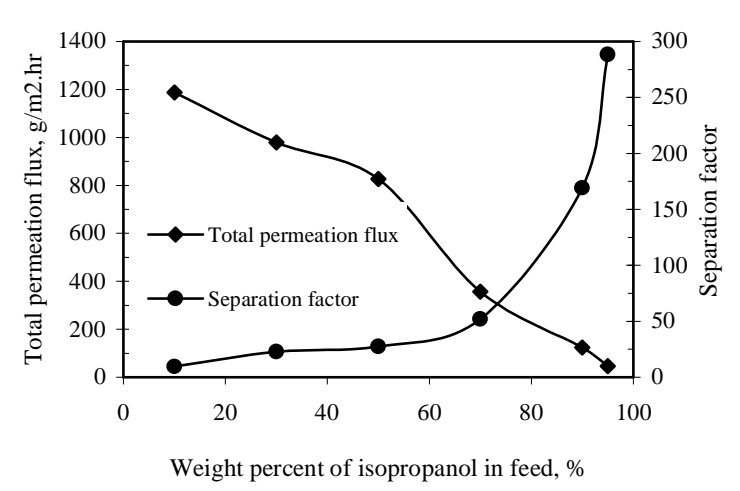

Fig. 6: PSI of the membranes at different $\%$ of isopropanol in feed.

However, at very high and low isopropanol content in the feed solution, there was no significant difference of PSI between the homogeneous and blended membranes. This was due to the high separation factor being compensated by a low permeation flux or vice versa. The above results indicated that the polymer blending technique especially at blend ratio of 30:70 (CS:PVA) had the ability to improve the overall pervaporation performances of chitosan membrane for the pervaporation dehydration of isopropanol and water mixtures.

\section{CONCLUSION}

The blend membranes were successfully prepared from chitosan and poly (vinyl alcohol) via a solution casting methods. Both the blended solutions and the formed membranes were optically clear and transparent. These revealed that the blends of chitosan and poly(vinyl alcohol) were compatible. Among the prepared blend ratios, the membranes with the blend ratio of 30:70 (CS/PVA) showed the highest separation factor for pervaporation dehydration of isopropanol/water mixture with 90 wt.\% of isopropanol in deed solutions. The blend membranes could improve the overall pervaporation performance, in term of pervaporation separation index (PSI), the membranes with the blend ratio of 30:70 (CS:PVA) always showed higher PSI than that of homogeneous membranes. However, both types of the membranes, homogeneous and blended were capable of dehydration.

\section{ACKNOWLEDGEMENTS}

The financial support provided for this research by the Ministry of Science, Technology and Environment of Malaysia through IRPA project is gratefully acknowledged. 


\section{REFERENCES}

[1] R.Y.M. Huang and W. Rhim (1993). "Separation Characteristics of Pervaporation Membrane Separation Process Using Modified Poly(vinyl alcohol) Membranes.” Polymer International. 30. 123-128.

[2] E. Ruckenstein and L. Liang (1996). "Poly(acrylic acid)Poly(vinyl alcohol) Semi- and Interpenetrating Polymer Network Pervaporation Membranes.” Journal of Applied Polymer Science. 62. 973-987.

[3] H.E.A. Brüschke (1990). "Removal of Ethanol from Aqueous Streams by Pervaporation." Desalination. 77. 323-329.

[4] I. Maeda and M. Kai (1991). "Recent Progress in Pervaporation membranes for water/ethanol separation." in Huang, R.Y.M. "Pervaporation Membrane Separation Processes." The Netherlands: Elsevier, Amsterdam. 111180.

[5] R.Y.M. Huang, FCIC and X. Feng (1993). "Pervaporation Separation Processes: History, Development and Separation Applications." Canadian Chemical News. March. 21-23.

[6] A. L. Andrady, A. Torikai and T. Kobatake (1996). "Spectral Sensitivity of Chitosan Photodegradation." Journal of Applied Polymer Science. 62. 1465-1471.

[7] J. K. Yang, I. L. Shih, Y. M. Tzeng, and S. L. Wang (2000), "Production and Purification of Protease from a Bacillus Subtilis that can Deproteinize Crustacean Wastes." Enzyme and Microbial Technology. 26. 406413.

[8] K.D. Yao, T. Peng, H.B. Feng and Y.Y. He (1994). "Swelling Kinetic and Release Characteristic of Crosslinked Chitosan: Polyether Polymer Network (Semi-IPN) Hydrogels.” Journal of Polymer Science: Part A: Polymer Chemistry. 32. 1213-1223.

[9] D.R. Paul, J.W. Barlow and H. Keskkula (1985). "Polymer Blends" in Mark,Bikales, Overberger and Menges. "Encyclopedia of Polymer Science." USA: John wiley \& Sons. 398-461.

[10] L. Fang and Goh, S. H. (2000). "Miscible Chitosan/Tertiary Amide Polymer Blends." Journal of Applied Polymer Science. 76. 1785-1790.

[11] H. S. Blair, J. Guthrie, T. K. Law and P. Turkington, (1987). "Chitosan and Modified Chitosan Membranes I. Preparation and Characterisation." Journal of Applied Polymer Science. 33. 641-656.

[12] L. G. Wu, C. L. Zhu, and M. Liu (1994). "Study of a new pervaporation membrane Part 1. Preparation and characteristics of the new membrane." Journal of Membrane Science. 90. 199-205.

[13] X. Chen, W. Li, Z. Shao, W. Zhong and T. Yu (1999). "Separation of Alcohol-Water Mixture by Pervaporation Through a Novel Natural Polymer Blend MembraneChitosan/Silk Fibroin Blend Membrane." Journal of Applied Polymer Science. 73. 975-980.

[14] A. Chanachai, R. Jiraratanano, D. Uttapap, G.Y. Moon, W.A. Anderson and R.Y.M. Huang (2000). "Pervaporation with chitosan/hydroxythelcellulose blended membranes." Journal of Membrane Science. 166. 271-280

[15] P. Sampranpiboon, R. Jiraratananon, D. Uttapap, X. Feng and R.Y.M. Huang (2000). "Pervaporation separation of ethyl butyrate and isopropanol with polyether block amide (PEBA) membranes." Journal of Membrane Science. 173. 53-59.

[16] M.G. Mohd. Nawawi, S. H. Tan, and H. Hassan (2001). "Pervaporation separation of Isopropanol-water mixtures using chitosan based membranes." 15th Symposium of Malaysian Chemical Engineers SOMChE 2001, 115-120,

[17] G. A. F. Roberts (1992). “Chitin Chemistry.” 1st ed. London: The Macmillan Press Ltd. 1-53.

[18] J. P. Brun, C. Larchet, G. Bulvestre and B. Auclair (1985). "Sorption and pervaporation of dilute aqueous solutions of organic compounds through polymer membranes." Journal of Membrane Science. 25. 55-100.

\section{NOMENCLATURE}

$\begin{array}{ll}\alpha & \text { Separation factor } \\ y_{A} & \text { Weight fraction of component A in permeate } \\ y_{\mathrm{B}} & \text { Weight fraction of component B in permeate } \\ x_{A} & \text { Weight fraction of component A in feed } \\ x_{B} & \text { Weight fraction of component B in feed } \\ \text { PSI } & \text { Pervaporation separation index, }\left(\mathrm{kg} / \mathrm{m}^{2} \cdot \mathrm{hr}\right) \\ J & \text { Total permeation flux, }\left(\mathrm{kg} / \mathrm{m}^{2} . \mathrm{hr}\right)\end{array}$

\section{BIOGRAPHY OF THE AUTHORS}

Tan Soon Huat received his B.Eng. (Chemical Engineering) and M.Sc. (Chemical Engineering) from Universiti Teknologi Malaysia (UTM) in 1999 and 2002, respectively. His research interest is in developing new types of polymeric membranes for liquid-liquid system and pervaporation process. Currently he is an academic staff with the School of Chemical Engineering, Universiti Sains Malaysia.

Associate Prof. Dr. Abdul Latif Ahmad is currently the Deputy Dean (Research and Postgraduate Study) at the School of Chemical Engineering, Engineering Campus, Universiti Sains Malaysia, Nibong Tebal, Penang. He was born in Selangor, Malaysia in 1967. He received his B.Eng. (Chemical Engineering), M.Sc. (Chemical Engineering) and Ph.D (Membrane Technology), all from the University of Wales, United Kingdom in 1990, 1991, and 1995 respectively. His area of research interest is membrane separation technology; synthesis and characterisation toward the application of environment monitoring and pollution control including water, wastewater and gas separation.

Dr. Mohd. Ghazali Mohd. Nawawi received his Bachelor's degree in Chemical Engineering from the New Mexico State University, USA in 1987. He obtained his master degree in Chemical Engineering from University College of Swansea, UK in 1990 and then graduated with a Ph.D degree from the University of Waterloo, Canada in 1997. He is currently a senior lecturer at Universiti Teknologi Malaysia. $\mathrm{He}$ is the Head of Separation Science and Engineering Research Group (SENSER). His research works focus on the 
development of natural polymeric membranes for the dehydration of alcohols using pervaporation.

Hashim Hassan graduated from Universiti Teknologi Malaysia (UTM) with a B. Eng. in Chemical Engineering in 1988. After graduation, he gained experience in membrane technology as research officer at Universiti Kebangsaan Malaysia (UKM). He received his M. Sc. (Chemical Engineering) in 1994, his thesis was on the production of hollow fiber membrane for gas separation. He joined UTM as a tutor in the Chemical Engineering Department in 1994. His research interest is in synthesis and preparation of novel membranes for pervaporation including the development of a mathematical model. 